\title{
Transient Clouding of a Sutureless Scleral Fixated Hydrophilic Intraocular Lens with Spontaneous Resolution: A Case Report and in vitro Experimental Test
}

\author{
Carla Danese $^{a, b}$ Renzo Calabresic Paolo Lanzetta ${ }^{a}, b, d$ \\ aDepartment of Medicine - Ophthalmology, University of Udine, Udine, Italy; ${ }^{b} \mathrm{Clinica}$ \\ Oculistica, Azienda Sanitaria Universitaria Friuli Centrale, Udine, Italy; 'Soleko IOL Division, \\ Pontecorvo, Italy; distituto Europeo di Microchirurgia Oculare - IEMO, Udine, Italy
}

\section{Keywords}

Cataract surgery $\cdot$ Hydrophilic intraocular lens $\cdot$ In vitro test $\cdot$ Intraocular lens $\cdot$ Transient intraocular lens clouding

\begin{abstract}
A 83-year-old woman underwent phacoemulsification in the left eye, complicated by posterior capsular rupture and dislocation of nuclear fragments in the vitreous cavity. She was left aphakic and referred to the Clinica Oculistica, Azienda Sanitaria Universitaria Friuli Centrale, Udine, Italy. After 2 days, she underwent pars plana vitrectomy, with removal of the nuclear fragments, and secondary intraocular lens (IOL) implantation. The lens used was a sutureless scleral fixation Carlevale lens (I71 FIL SSF Carlevale lens ${ }^{\mathrm{TM}}$. Soleko IOL Division), which was delivered to the operating theatre on the morning of the operation. During the implantation, it appeared opaque, without any deposit on its surfaces. The lens was not removed. On the next day, the lens was transparent, and it remained clear at the following control visits. An in vitro experimental test demonstrated that $25 \%$ hydrophilic polymers are vulnerable to thermal shock, developing an opacification which resolves after temperature stabilization of the lens.
\end{abstract}

\section{Introduction}

Intraocular lens (IOL) opacification is a recognised complication of cataract surgery: indeed, a recently published study showed that the first cause of IOL explantation is opacification or discoloration of the optic. This usually occurs after $>5$ years since cataract surgery, and it is almost always caused by superficial and subsurface deposits of calcium phosphate, 
Danese et al.: Transient Clouding of Hydrophilic Intraocular Lens

most frequently on hydrophilic IOLs [1]. However, there are few cases in literature reporting a transient IOL opacification involving both hydrophilic and hydrophobic lenses occurring intraoperatively and resolving few hours after intervention [2-6]. From 2018 to July 2020, 18 Carlevale IOLs (I71 FIL SSF Carlevale lens ${ }^{\mathrm{TM}}$. Soleko IOL Division) have been implanted in our centre, mainly due to aphakia and IOL subluxation. In all cases, surgery was uneventful with a centered and stable implantation. In 1 case, we observed an intraoperative transient opacification of the newly implanted IOL. This case report describes this rare event which resolved without any permanent sequelae.

\section{Case Report}

A 83-year-old woman underwent cataract surgery in the left eye. She was pseudophakic in the right eye. The operation was complicated by posterior capsule rupture and displacement of nuclear fragments into the vitreous chamber. After anterior vitrectomy was performed, surgery was terminated and she was left aphakic. On the next day, she was referred to our centre for vitrectomy, nucleus remnants removal, and secondary IOL implantation. Her visual acuity was 20/200 and the eye presented mild corneal oedema, Descemet's folds, and nuclear fragments in the vitreous chamber. The surgeon (PL) scheduled surgery for the following day due to the lack of an adequate IOL for implantation. A +28 diopter foldable sutureless scleral fixation Carlevale IOL was chosen. This is a single-piece $25 \%$ water content hydrophilic acrylic IOL with 2 anchor optics on either side that are fixated transclerally. The IOL was ordered from the supplier. The morning of the planned surgery the IOL was delivered the operating room with a travel time of approximately $90 \mathrm{~min}$. The external temperature was $-1^{\circ} \mathrm{C}$. Presumably, the temperature within the vehicle of the shipper was higher: The IOL was stored in the operating room at $19^{\circ} \mathrm{C}$ one hour before implantation. Under peribulbar anaesthesia, a 25-gauge pars plana vitrectomy was performed uneventfully, with successful removal of the nuclear fragments from the vitreous chamber. Immediately after removing the IOL from its package, before folding it inside the injector, it presented an altered colouring to milky white of the central optic which was initially attributed to light reflection under the microscope (shown in Fig. 1a). Once the IOL was implanted into the eye it showed a white colouring of the central optic which tended to increase to dense white at the end of surgery. A decision not to explant the IOL was taken after contacting the supplier, who ascribed the opacification to temperature changes and stated that clouding was temporary. After implantation further procedures in the vitreous chamber and visualization of the fundus were not significantly impaired due to IOL opacity. The discoloration was located in the body of the optic plate, in absence of surface opacities. After careful evaluation, using a pupillary expansion ring to achieve a better visualization of the extension of the opacity the operation was terminated without removing the lens (shown in Fig. 1b). On the next day, after pharmacological mydriasis, the optic of the IOL appeared entirely transparent (shown in Fig. 1c). These findings were confirmed also on the next visit 1 week after the operation when the IOL was optically transparent and well positioned. Three months after the operation, the IOL did not change appearance, and the corrected distance visual acuity was 20/25.

\section{In vitro Experiment}

In order to achieve a better understanding of the effect of a thermal change on a $25 \%$ hydrophilic IOL, an in vitro experimental test was conducted at Soleko IOL division laboratories. To simulate the temperature change, an IOL composed of the same material as the 1 implanted in the present case report was immersed in water and kept for $5 \mathrm{~h}$ in a cold room, at a temperature of $5^{\circ} \mathrm{C}$. Subsequently, it was placed in an oven, at the temperature of $36^{\circ} \mathrm{C}$.

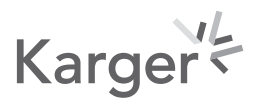




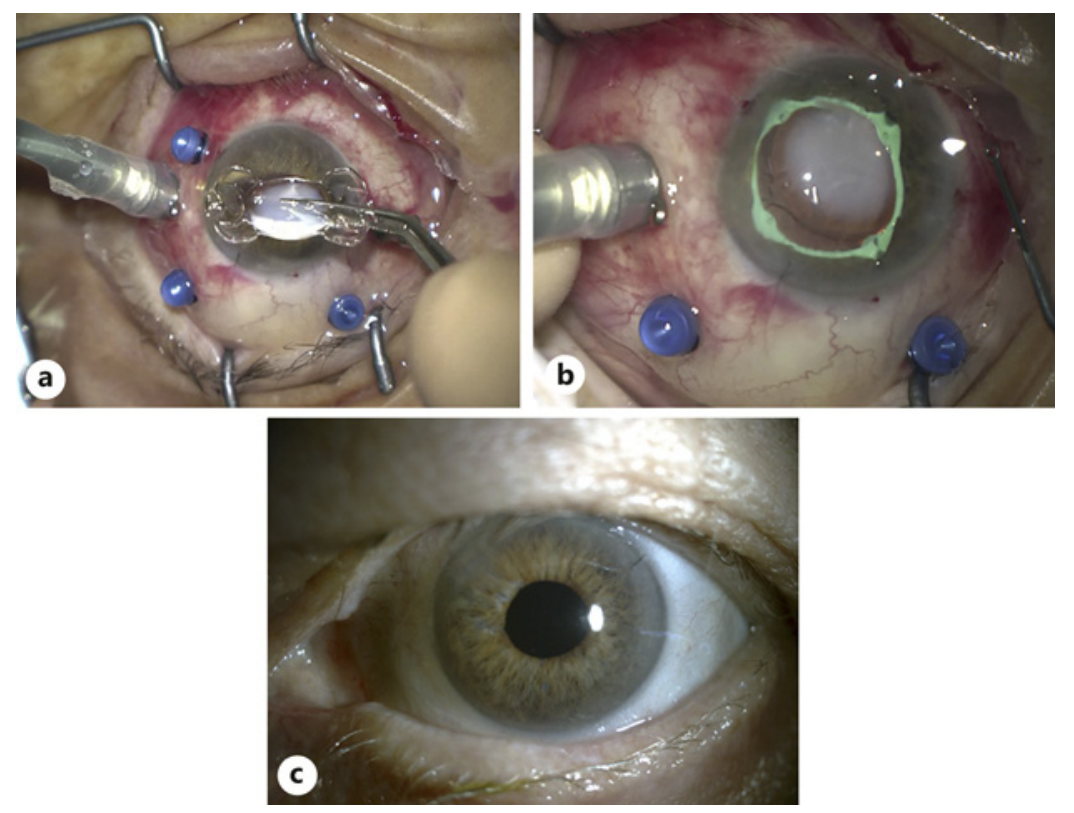

Fig. 1. a Before implantation, the optic plate of the IOL appeared milky white. $\mathbf{b}$ The pupillary expansion ring allowed better visualization of the dense white opacity. c On the first post-operative day, the optic plate of the IOL appeared transparent. IOL, intraocular lens.

This resulted in an immediate opacification of the IOL surface. The IOL was kept at constant temperature, and the cloudiness progressively reduced in dimensions, until it disappeared after $3 \mathrm{~h}$ (shown in Fig. 2; online suppl. video 1; for all online suppl. material, see www.karger. com/doi/10.1159/000510930).

\section{Discussion}

Transient intraoperative IOL opacification has been described previously. McKibbin et al. [2] reported acute, transient clouding of 2 hydrophobic IOLs after they were heated at $47^{\circ} \mathrm{C}$ before implantation. Differently, Zhang et al. [5] and Sonbolestan and Abtahi [6], described the same phenomenon after hydrophilic lenses were kept at -7 and $-10^{\circ} \mathrm{C}$, respectively. Similarly, Tyagi et al. [3] and Helvaci [4] report the clouding of both hydrophilic and hydrophobic lenses after they were stored in a cold room and were delivered to the operating room shortly before surgery. However, no data about the exact external temperature are available $[3,4]$.

The most likely mechanisms correlated to transient cloudiness of the lens seem to be thermic shock and transient physical changes of the material and refraction properties. Therefore, acute opacification may occur due to rapid temperature changes.

This is the first time that an experimental test has been conducted in order to study the phenomenon. The experiment demonstrated that $25 \%$ hydrophilic polymers are susceptible to temperature changes, which leads to clouding of the IOL material. However, the test also highlighted that the opacification is transient: indeed, it disappears keeping the IOL at room temperature.

The case of the patient described in the present report is also peculiar because the thermal excursion was presumably limited as compared to those evidenced in the previous studies. Moreover, IOL opacification was evident before implantation into the eye and progressed after scleral fixation. 

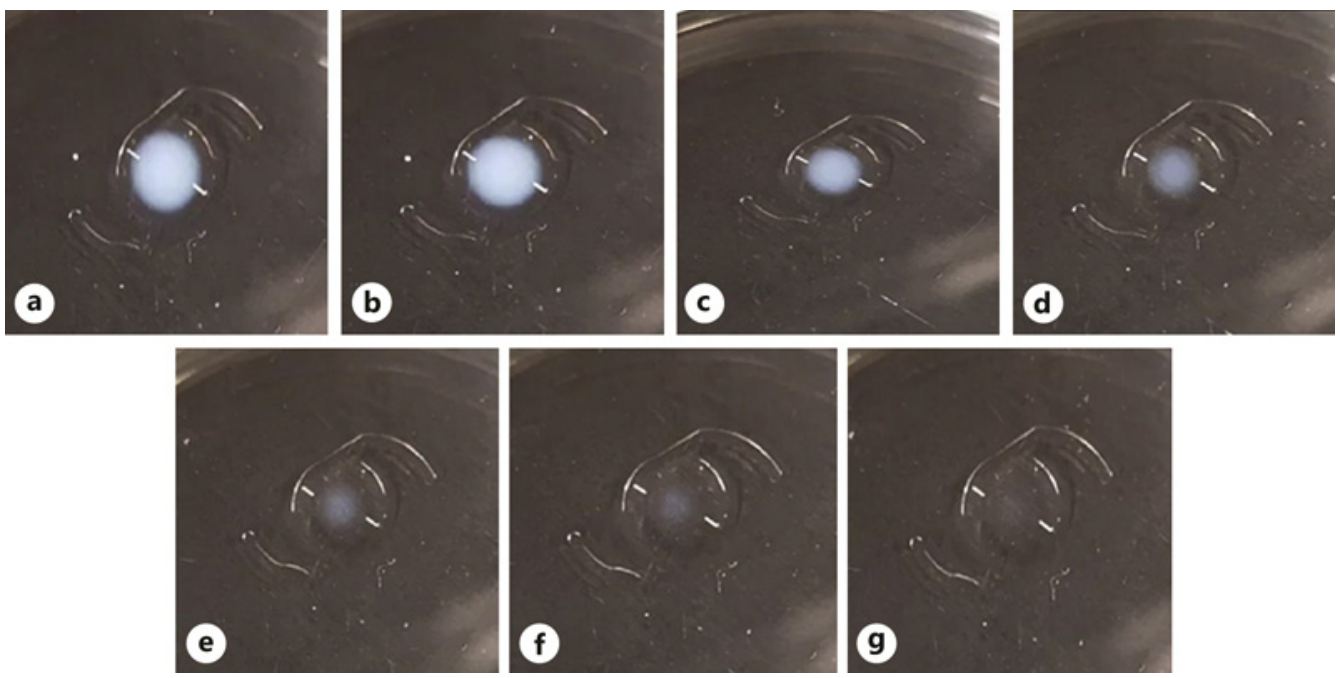

Fig. 2. Results of the in vitro experimental test. a Opacification of the IOL at the moment of the termic shock. b-g Pictures showing the resolution of the clouding with the IOL being kept at constant temperature of $36^{\circ} \mathrm{C}$ (half an hour (b), one hour (c), one and a half hour (d), two hours (e), two and a half hours (f), three hours (g)). IOL, intraocular lens.

To our knowledge, this is the first reported case of intraoperative transient IOL opacification which occurred before lens implantation. Also, this is the first description of opacification of a scleral fixation hydrophilic lens occurring during secondary IOL implantation. The in vivo observations have been supported and confirmed with an in vitro experiment.

In the rare event that this complication may occur intraoperatively after IOL implantation, it is advisable that the surgeon does not proceed with a subsequent explantation because the opaque lens will likely return to a transparent status within few hours after surgery. Based on the above-mentioned evidence, this option is also valid in the case that cloudiness is noticed before implantation and temperature fluctuation of the environment where the lens is stored is assessed.

\section{Statement of Ethics}

This work has been performed according to the guidelines for human studies and has been performed ethically in accordance with the World Medical Association Declaration of Helsinki. The subject has given informed consent to publish the case, including publication of images. Information revealing the subject's identity has been avoided. The patient is not identified by the real name.

\section{Conflict of Interest Statement}

Carla Danese: none. Renzo Calabresi: Soleko, E. Paolo Lanzetta: Bayer, C; Centervue, C; Novartis Pharma AG, C. 


\section{Funding Sources}

The authors did not receive any funding.

\section{Author Contributions}

Carla Danese: acquisition of data for the work and drafting the work. Renzo Calabresi: acquisition of data for the work and revising the work critically for important intellectual content. Paolo Lanzetta: design of the work, interpretation of data for the work and revising the work critically for important intellectual content. All the authors: final approval of the version to be published and agreement to be accountable for all aspects of the work in ensuring that questions related to the accuracy or integrity of any part of the work are appropriately investigated and resolved.

\section{References}

1 Neuhann T, Yildirim TM, Son HS, Merz PR, Khoramnia R, Auffarth GU. Reasons for explantation, demographics, and material analysis of 200 intraocular lens explants. J Cataract Refract Surg. 2020;46(1):20-6.

2 McKibbin M, Seemongal-Dass RR, Atkinson PL. Transient fogging of acrylic (Acrysof) intraocular lenses. Eye. 1999;13(Pt 5):672-3.

3 Tyagi P, Shah N, Jabir M. Intraoperative clouding of a posterior chamber intraocular lens. Int Ophthalmol. 2011;31(6):483-4.

4 Helvaci S. Acute opacification of hydrophobic acrylic intraocular lens during implantation: result of temperature variation. Arq Bras Oftalmol. 2015;78(4):267.

5 Zhang T, Li S, Liu C, Zhao R, Chang C, Han N. Acute clouding of a trifocal intraocular lens with spontaneous resolution: a case report. BMC Ophthalmol. 2019;19(1):208.

6 Sonbolestan SA, Abtahi ZA. Transient intraocular lens opacification during phacoemulsification surgery. J Curr Ophthalmol. 2019;31(3):342-4. 